\title{
Modified Commutators vs Modified Operators in a Quantum Gravity minimal length scale
}

\author{
Joey Contreras ${ }^{1, \mp *}$ Michael Bishop ${ }^{2, \ddagger}$ and Jaeyeong Lee ${ }^{3, \ddagger}$ and Douglas Singleton ${ }^{4, \ddagger}$ \\ 1 Physics Department, California State University Fresno, Fresno, CA 93740; mkfetch@mail.fresnostate.edu \\ 2 Mathematics Department, California State University Fresno, Fresno, CA 93740; \\ mibishop@mail.fresnostate.edu \\ 3 Physics Department, California State University Fresno, Fresno, CA 93740; yeong0219@mail.fresnostate.edu \\ 4 Physics Department, California State University Fresno, Fresno, CA 93740; dougs@mail.fresnostate.edu \\ * Correspondence: mkfetch@mail.fresnostate.edu; Tel.: +1-559-278-2371 (U.S.A) \\ $\ddagger \quad$ These authors contributed equally to this work.
}

Citation: Contreras, J.; Bishop, M.; Lee, J.; Singleton, D. Modified Commutators vs Modified Operators in a Quantum Gravity minimal length scale. Proceedings 2021, 1, 0 .

https://doi.org/

Received:

Accepted:

Published:

Publisher's Note: MDPI stays neutral with regard to jurisdictional claims in published maps and institutional affiliations.

Copyright: () 2020 by the authors. Licensee MDPI, Basel, Switzerland. This article is an open access article distributed under the terms and conditions of the Creative Commons Attribution (CC BY) license (https: / / creativecommons.org / licenses/by/4.0/).

\begin{abstract}
Generic theories of quantum gravity often postulate that at some high energy/momentum scale there will be a fixed, minimal length. Such a minimal length can be phenomenologically investigated by modifying the standard Heisenberg Uncertainty relationship. This is generally done in practice by modifying the commutator between position and momentum operators, which in turn means modifying these operators. However, modifications such that the uncertainty relation changes lead to conflicts with observational data (gamma ray bursts). This arises in the form of a predicted minimal length energy scale that is above the Planck energy rather than below it. As a result there seems to be an implication that there is no minimal length scale in these generic theories. Meanwhile, modifying the operators such that the standard uncertainty relation retains the same form, leads to no such conflict with observational data. We show that it is this modification of the position and momentum operators that is the key determining factor in the existence (or not) of a minimal length scale. By focusing primarily on the role of these operators we also show that one can avoid the constraints from the observations of short gamma ray bursts, which in certain cases seem to push the minimal length scale above the Planck scale.
\end{abstract}

Keywords: High energy; quantum gravity; minimal length; generalized uncertainty principle

\section{Introduction}

Quantum gravity models often have the idea of a minimal distance at high energy/momentum scales [1]. There have been several proposals to test for a minimal length in a laboratory setting [2] or using the detection of gravitational waves [3]. The most stringent constraints on a minimal length scale come from astrophysical observations related to the propagation of photons through long distances in spacetime. One particular proposal [4-6] is to test for a minimal length scale using observations of gamma ray bursts (GRB). Gamma ray bursts are emissions of extremely high energy photons that generally are detected after traveling cosmological distances. Gamma ray bursts fall into two categories: (i) short gamma ray bursts which are thought to come from neutron star mergers or neutron star-black hole mergers and (ii) long gamma ray bursts which are thought to come from supernova. Short gamma ray bursts are most useful for observing the effects of a minimal length scale. Models predict that photons detected from GRBs should exhibit dispersion due to the existence of a minimal length i.e. photons of different energies will have slightly different velocities when traversing spacetime.

In references [4-6] a generic quantum gravity modified energy-momentum relation for photons was proposed of the form

$$
p^{2} c^{2}=E^{2}\left[1+f\left(E / E_{Q G}\right)\right],
$$


where $f\left(E / E_{Q G}\right)$ is some arbitrary function associated with one's theory of quantum gravity and with $E_{Q G}$ being the energy scale of quantum gravity. Often this is set to be the Planck scale $\left(E_{Q G}=E_{P l}=\sqrt{\hbar c^{5} / G} \approx 10^{19} \mathrm{GeV}\right)$. When $E \ll E_{Q G}$, the Taylor expansion of (1) leads to

$$
p^{2} c^{2}=E^{2}\left[1+\xi\left(E / E_{Q G}\right)+\mathcal{O}\left(E / E_{Q G}\right)^{2}\right] .
$$

The sign of $\xi$ is model dependent. From Hamilton's equation, the photon's velocity is given as $\frac{\partial E}{\partial p}=v$. Using (2) and series expanding gives an energy dependent photon velocity

$$
v=\frac{\partial E}{\partial p} \approx c\left(1-\xi \frac{E}{E_{Q G}}\right) .
$$

This energy dependence of photon velocity leads to a difference in the arrival time, $\delta t$, of the photons with energy difference $\delta E$. To first order in $E$ this time delay is

$$
\delta t=\xi \frac{L}{c} \frac{\delta E}{E_{Q G}},
$$

where $L$ is the distance the photons traveled. The implication is that farther distances and larger energy differences will lead to bigger time delays between photons. A goal of this work however is to show that it is possible to have a minimal length scale while avoiding this dispersion.

In 2009 [7], the Fermi Gamma-Ray space telescope detected a short GRB (GRB090510). This observation allowed one to constrain the quantum gravity scale from 1.2 to 100 times the Planck scale depending on whether one made conservative or liberal assumptions (i.e. $M_{Q G} \geq 1.2 M_{P l}$ to $\left.M_{Q G} \geq 100 M_{P l}\right)$. One of the assumptions that went into setting these bounds is that the lowest order correction to the dispersion relationship from (1) was linear in $E$ as in (2). If the lowest order correction is of order $E^{2}$ or higher then the constraints are much weaker. This result appeared to contradict the expectation that one should find evidence for a minimal length scale well before reaching the Planck scale. If taken at face value these results present a challenge to the ideas of minimal distance scales emerging from quantum gravity.

Modified dispersion relationships of the form in (1) can be obtained from modifications of special relativity called deformed special relativity or double special relativity (DSR) [5,6,8-11]. DSR contains the idea of an observer independent minimal length. In this work we will approach a minimal length through a modification of the quantum mechanical position and momentum operators and their commutator. This approach is known as the generalized uncertainty principle (GUP) [12].

In this paper we present a minimal length scale model which does not lead to an energy dependent speed of light and thus avoids the constraints of the GRB observations. Modified position and momentum operators are used which lead to a minimal length that is consistent with observation. This can be accomplished with or without modifying the canonical commutation relationship.

\section{Modified energy-momentum relationship without photon dispersion}

We propose modified momentum operators which do not lead to dispersion of photons while at the same time, maintaining a minimal length scale. Two variants of modified momentum operators are

$$
p^{\prime}=p_{0} \tanh \left(\frac{p}{p_{0}}\right) \quad \text { and } \quad p^{\prime}=p_{0} \arctan \left(\frac{p}{p_{0}}\right) .
$$

These two modified momentum operators have the common feature of being bounded and going over to the ordinary momentum at small $p$ (i.e. $p^{\prime} \rightarrow p_{0}$ as $p \rightarrow \infty$ and $p^{\prime} \rightarrow p$ for $p \ll p_{0}$ ). The parameter $p_{0}$ is the maximum momentum which characterizes the quantum gravity scale; its connection to minimum distance is shown in the following section. This 
idea of modified momentum operators having an upper bound is loosely motivated by Born-Infeld electrodynamics [13] where the well known Maxwell's equations are modified so as to have a maximum electric field strength.

In Minkowski spacetime (flat with no curvature), one gets the dispersion relationship for a particle of mass $m$ from the energy-momentum relation $E^{2}=p^{2} c^{2}+m^{2} c^{4}$. For massless particles this energy-momentum relationship becomes $E=p c$. Using Hamilton's equation one finds $\frac{\partial E}{\partial p}=c$ so that the velocity does not depend on $E$ or $p$ and there is no dispersion. For the modified momenta, $p^{\prime}$, in (5) we want to write down an associated modified energy, $E^{\prime}$, so that in terms of $p^{\prime}$ and $E^{\prime}$ one has the standard relationship $E^{\prime}=p^{\prime} c$. Then by Hamilton's equation in terms of these modified energy and momentum one finds $\frac{\partial E^{\prime}}{\partial p^{\prime}}=c$ and there is no dispersion. Since the modified momentum $p^{\prime}$ are bounded by $p_{0}$ the modified energy should also bounded by a maximum energy $E_{0}$. As $p \rightarrow \infty$ we want a modified energy that satisfies

$$
E^{\prime}=p^{\prime} c \rightarrow E_{0}=p_{0} c \text { as } p \rightarrow \infty .
$$

Using the two modified momentum operators from (5) in the energy-momentum relationship of (6) one finds that the modified energies are

$$
E^{\prime}=p_{0} c \tanh \left(\frac{p}{p_{0}}\right)=E_{0} \tanh \left(\frac{E}{E_{0}}\right)
$$

and

$$
E^{\prime}=p_{0} c \arctan \left(\frac{p}{p_{0}}\right)=E_{0} \arctan \left(\frac{E}{E_{0}}\right) .
$$

To obtain the last expressions in (7) and (8) we used $p=\frac{E}{c}$ and $p_{0}=\frac{E_{0}}{c}$.

The energy-momentum relationship between the modified energy and modified momentum as given in equations (5), (7), and (8) is the same as the standard energymomentum relationship. These modified energies and momenta do not lead to dispersion since the energy-momentum relationship in (6) is unchanged. This lack of dispersion can be seen either from the phase velocity, $\frac{E^{\prime}}{p^{\prime}}=c$, or the group velocity obtained via Hamilton's equation, $\frac{\partial E^{\prime}}{\partial p^{\prime}}=c$. In contrast, several common phenomenological models of quantum gravity $[4-6,8,9]$ give a modified energy-momentum relationship, like that in (1) and (2), which leads to photon dispersion as in equation (3).

\section{Minimal length via modified operators}

A generalized uncertainty principle is often used as an approach to a minimum length scale in phenomenological quantum gravity.[12,14-19] Here a common approach where one adds a term $\beta \Delta p^{2}$ to the usual Heisenberg relationship is reviewed.

$$
\Delta x \Delta p \geq \frac{\hbar}{2} \rightarrow \Delta x^{\prime} \Delta p \geq \frac{\hbar}{2}\left(1+\beta \Delta p^{2}\right) .
$$

The phenomenological parameter $\beta$ is used to characterize the effects of quantum gravity while the primed quantities represent the modified operators. Only the position operator is modified in this particular approach. 


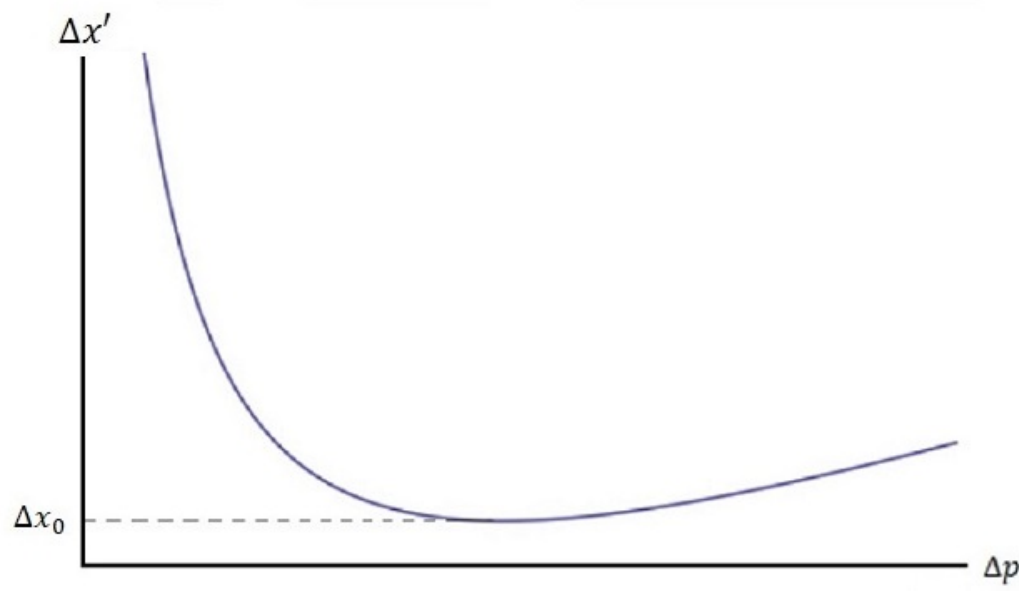

Figure 1. Generalized Uncertainty Principle from equation (9) where $\Delta x^{\prime}$ has a local minimum of $\Delta x_{0}=\hbar \sqrt{\beta}$ at $\Delta p=\frac{1}{\sqrt{\beta}}$

For the canonical Heisenberg uncertainty relationship $\Delta x \propto \frac{1}{\Delta p}$, so as $\Delta p \rightarrow \infty$ one has $\Delta x \rightarrow 0$ i.e. there is no absolute, minimum length. However, from (9) consider that $\Delta x^{\prime}=\frac{\hbar}{2}\left(\frac{1}{\Delta p}+\beta \Delta p\right)$. Now it can be demonstrated that $\Delta x^{\prime}$ has a local minimum, occurring at $\Delta p=1 / \sqrt{\beta}$, which gives a minimum length of $\Delta x_{0}=\hbar \sqrt{\beta}$ as shown in Fig. 1 .

The justification of adding the $\beta \Delta p^{2}$ term in (9) goes as follows: as distances become shorter and shorter via higher energy collisions, at some point the center of mass energy will become great enough to create a micro black hole. This black hole will have a Schwarzschild radius set by the mass-energy in the collision i.e. $\Delta x \sim R_{s c h} \sim E$. As one goes to even higher energy/momentum scales in the collision the resulting Schwarzschild radius will grow linearly which will prevent one from probing smaller distances at the threshold.

Having a modified uncertainty relationship like (9) implies a modification of the commutator between the position and momentum operators. The usual commutator $[\hat{x}, \hat{p}]=i \hbar$ leads to the following connection with the HUP

$$
\Delta x \Delta p \geq \frac{1}{2}|\langle[\hat{x}, \hat{p}]\rangle| \rightarrow \Delta x \Delta p \geq \frac{\hbar}{2} .
$$

A GUP like that in (9) implies a modified commutator of the form

$$
\left[\hat{x}^{\prime}, \hat{p}\right]=i \hbar\left(1+\beta p^{2}\right) .
$$

In [12], the position operator was modified while the momentum operator was not, and this is the reason that the $\Delta p$ and $\hat{p}$ are not primed in (9) and (11).

Modifying the commutator as in (11) comes with some challenges as reviewed in [20-23]. These challenges are: (i) violation of the equivalence principle; (ii) the "soccer" ball problem [24] (i.e. the difficulty in constructing consistent multi-particle states); (iii) velocity dependence of position and momentum uncertainties. In the present work we avoid these problems as well as the constraints coming from GRB observations. We do this by modifying the position and momentum operators so as to give a minimal length, while at the same time preserving the usual commutator and usual energy-momentum relationship for these modified operators.

The usual inference drawn from the above arguments is that modification of the commutator determines the existence of a minimal distance. However, it was shown in reference [25] that this is not necessarily the case; there are a host of different ways to alter the position and/or momentum operators while leading to the same commutator (11) and these may or may not lead to a minimal length. If instead of (9) one modifies the momentum operator then one would find that $\Delta x^{\prime} \Delta p^{\prime} \geq \frac{\hbar}{2}\left(1+\beta \Delta p^{2}\right)$. In this case $\Delta x^{\prime}$ would be 
proportional to $1 / \Delta p^{\prime}+\beta \Delta p^{2} / \Delta p^{\prime}$, and whether or not this has a minimum would depend on the detailed behavior of $\Delta p^{\prime}$. The conclusion of [25] was that the existence of a minimal length is determined by the specific modification of the position and momentum operators rather than the way the commutator is modified.

Here we show that we can use the modified momentum operators of (5) to obtain a minimum length scale without modifying the form of the fundamental commutator. If the commutator's canonical form is to be preserved the position operator must be modified as well. In other words, we want to preserve the relationship

$$
\left[\hat{x}^{\prime}, \hat{p}^{\prime}\right]=i \hbar \rightarrow \Delta x^{\prime} \Delta p^{\prime} \geq \frac{\hbar}{2} .
$$

We refer to (12) as the Modified Heisenberg Uncertainty principle (MHUP) since the operators are modified but the commutation relationship is kept the same, unlike the GUP where the commutator is modified. For the usual HUP, written in terms of $\Delta x$ and $\Delta p$, there is no lower bound on $\Delta x$. This is because $\Delta x \propto \frac{1}{\Delta p}$ so $\Delta x \rightarrow 0$ as $\Delta p \rightarrow \infty$. However, for the modified momentum operators in (5), $\Delta p^{\prime}$ is bounded above by $p_{0}$ and so $\Delta x^{\prime}$ will be bounded below by $\Delta x^{\prime}>\frac{\hbar}{2 p_{0}}$. To see the upper bound on uncertainty in modified momentum, we note that the uncertainty of the modified momentum is $\Delta p^{\prime}=\sqrt{\left\langle p^{\prime 2}\right\rangle-\left\langle p^{\prime}\right\rangle^{2}}$. From $\left\langle p^{\prime}\right\rangle^{2} \geq 0$ one has $\Delta p^{\prime} \leq \sqrt{\left\langle p^{\prime 2}\right\rangle}$. Since the magnitude of the momentum operator $p^{\prime}$ is bounded above by $p_{0}$, we find $\left\langle p^{\prime 2}\right\rangle<p_{0}^{2}$ and thus $\Delta p^{\prime}<p_{0}$.

Fig. 2 shows the behavior of the MHUP as given by (12) with $\Delta p^{\prime}<p_{0}$ and thus $\Delta x^{\prime}>\frac{\hbar}{2 p_{0}}$. In comparing Fig. 1 with Fig. 2 one sees that although both have a non-zero minimum for $\Delta x^{\prime}$ the way in which the minimum is obtained is different. Modifying the position-momentum commutator as in (11) leads to a $\Delta x^{\prime} \Delta p^{\prime}$ graph which reaches a minimum $\Delta x^{\prime}$ and then increases with $\Delta p^{\prime}$ as shown in Fig. 1 . In the present work where the operators are modified, but the commutator retains its standard form, we find that this leads to a $\Delta x^{\prime} \Delta p^{\prime}$ graph which asymptotically approaches the non-zero, minimum value of $\Delta x^{\prime}$ with increasing $\Delta p^{\prime}$ as shown in Fig. 2. This is one of the differences between the prior GUP approaches and the present approach to a minimal distance. Other works [26] [27] have also looked at the possibility of having a minimal length while maintaining the standard commutator for modified position and momentum.

We need to modify the standard position operator which in momentum space is $\hat{x}=i \hbar \partial_{p}$ in order to preserve the standard commutator of (12). Assuming that our modified position operator has the form

$$
\hat{x}^{\prime}=i \hbar f(p) \partial_{p},
$$

we choose the function $f(p)$ so as to make (12) true for either modified momentum in (5). 


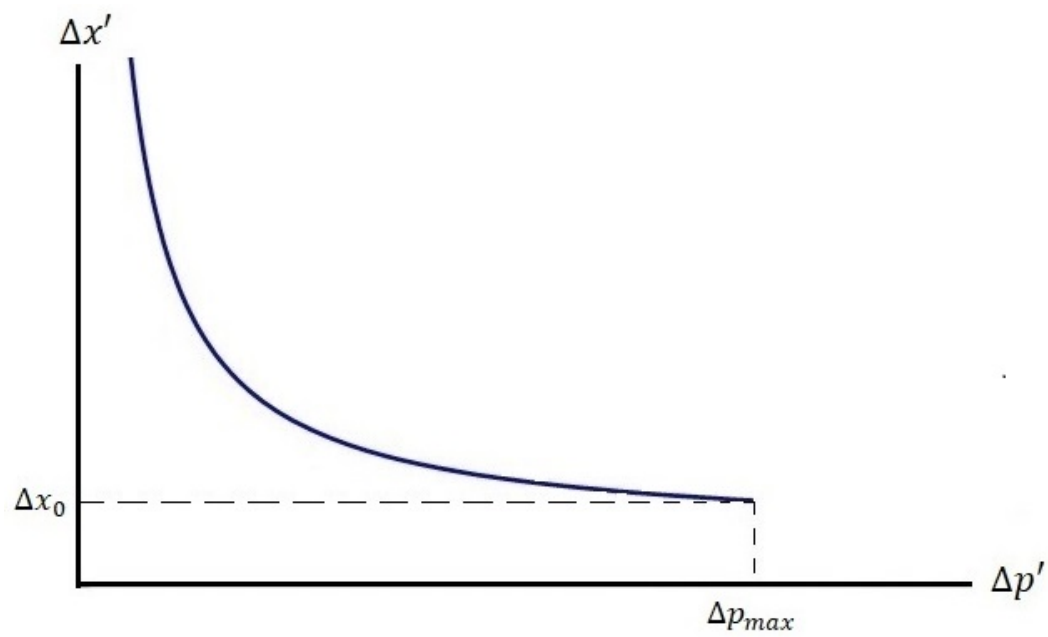

Figure 2. The relationship between $\Delta x^{\prime}$ and $\Delta p^{\prime}$ from the Modified Heisenberg Uncertainty Principle where $\Delta p_{\max }=p_{0}$ and $\Delta x^{\prime}>\frac{\hbar}{2 p_{0}}=\Delta x_{0}$.

Inserting the modified position (13) and modified momenta (5) into $\left[\hat{x}^{\prime}, \hat{p}^{\prime}\right]$ we find

$$
\left[\hat{x}^{\prime}, p_{0} \tanh \left(\frac{p}{p_{0}}\right)\right]=i \hbar f(p) \operatorname{sech}^{2}\left(\frac{p}{p_{0}}\right),
$$

and

$$
\left[\hat{x}^{\prime}, p_{0} \arctan \left(\frac{p}{p_{0}}\right)\right]=i \hbar f(p)\left(1+\left(\frac{p}{p_{0}}\right)^{2}\right)^{-1} .
$$

Requiring that the right hand sides of (14) and (15) equal it leads to the following modified position operators

$$
\hat{x}^{\prime}=i \hbar \cosh ^{2}\left(\frac{p}{p_{0}}\right) \partial_{p} \text { for } \hat{p}^{\prime}=p_{0} \tanh \left(\frac{p}{p_{0}}\right)
$$

and

$$
\hat{x}^{\prime}=i \hbar\left[1+\left(\frac{p}{p_{0}}\right)^{2}\right] \partial_{p} \text { for } \hat{p}^{\prime}=p_{0} \arctan \left(\frac{p}{p_{0}}\right) .
$$

Note that the position operator in (17) is that same as the modified position operator used in [12] if one sets $\beta=1 / p_{0}^{2}$. The modified position operator in (16) is reminiscent of that proposed in [28] but with $\cosh ^{2}\left(p / p_{0}\right) \rightarrow \operatorname{sech}^{2}\left(p / p_{0}\right)$.

We have found modified position operators, modified momentum operators (given in equations (16) and (17)), and modified energies (given in (7) and (8)) which preserve the usual position-momentum commutator and the usual energy-momentum relationship (of the same form). The modified operators lead to a minimum distance as illustrated in Fig. 2, while the energy-momentum relationship avoids the dispersion mentioned earlier. Additionally the problems [20-24] connected with the GUP are avoided.

In both (16) and (17) the position operator has been altered. It is in terms of these modified position operators, $\hat{x}^{\prime}$, that a minimum length as opposed to the usual position operator, $\hat{x}=i \hbar \partial_{p}$ where there is no such minumum length. This can be seen in Figs. 1 and 2 which plot $\Delta x^{\prime}$ versus $\Delta p^{\prime}$ rather than $\Delta x$ versus $\Delta p^{\prime}$. The question arises if this modified definition position and the related $\Delta x^{\prime}$ are physically meaningful. We approach this question from the angle of "Do the modified operators in (16) or (17) have a proper Hilbert space representation to allow them to be proper position and momentum operators?". The demonstration that modified position operators like those in (16) and (17) allow for the construction of a Hilbert space and satisfy the requirements to be physically meaningful 
position operators was carried out already in reference [12]. As already noted, the position operator in (17) is exactly the same as the modified position operator used in [12] but with $\frac{1}{p_{0}^{2}} \rightarrow \beta$. In regard to the physical reasonableness of these modified position operators in (16) and (17), we note that in the limit $p \ll p_{0}$ both of the modified $\hat{x}^{\prime} \mathrm{s}$ become the standard momentum operator. In the final section we give estimates on the lower bounds on $p_{0}$ coming from observations.

\section{Summary and Conclusions}

From the bottom up, phenomenological approaches to quantum gravity such as double special relativity $[8,9]$ will generically have the idea of an absolute minimal distance scale. Top down approaches to quantum gravity also incorporate this idea of a minimumal length scale, such as string theory or loop quantum gravity.

This absolute minimal length scale is generally considered to be around the Planck scale and thus difficult to test for. However, in [4] the proposal was made that one could test for this minimal length scale using short GRBs. This is due to the idea that the minimal distance scale would lead to an energy-dependent dispersion of gamma rays detectable through differences in arrival times of photons with different energies. Observations of one such GRB (GRB090510) by the Fermi-Gamma ray observatory [7] have placed constraints on this minimal distance scale to be sub-Planckian. This operated under the assumption that the relationship between energy and momentum was of the general form (1) and (2) which lead to an energy dependence of the photon's velocity as given in (3) as well as a time delay (4). This constraint also assumed that the lowest order correction to the photon velocity was linear in energy $E$.

In this work modified momenta was proposed, (5), as well as associated modified energies, (7) and (8) which had a maximum cut off (inspired by Born-Infeld electrodynamics [13] which include a maximum electric field). However, note the relationship between the modified momenta and energies, given in (7) and (8), differ from the relationships given in (1) and (2). The energy-momentum relationship in (7) and (8) once again do not lead to an energy-dependent photon velocity and thus no dispersion occurs. Thus the constraints coming from the GRB observations are avoided. In contrast the energymomentum relationship in (1) and (2) do in fact lead to an energy dependent photon velocity as given in (3).

We modified the position, momentum and energy operators in such a way that the standard position-momentum commutator and the standard energy-momentum relationship had the same forms as in canonical quantum mechanics and special relativity but in terms of the modified operators. One might ask what happens to Lorentz symmetry in the present case - in particular what happens to boost transformations. Consider the generators of boosts $x^{0} p-x p^{0}=(c t) p-x(E / c)$. Since the energy and momentum are modified in the same way - see (5), (7) and (8) - this implies that one should modify the time in the same way as position. For the the tanh modification this is

$$
\hat{x}^{0}=c \hat{t}^{\prime}=i \hbar \cosh ^{2}\left(\frac{p}{p_{0}}\right) \frac{\partial}{\partial p}=i \hbar c \cosh ^{2}\left(\frac{E}{E_{0}}\right) \frac{\partial}{\partial E},
$$

and for the arctan modification this is

$$
\hat{x}^{\prime 0}=c \hat{t}^{\prime}=i \hbar\left[1+\left(\frac{p}{p_{0}}\right)^{2}\right] \frac{\partial}{\partial p}=i \hbar c\left[1+\left(\frac{E}{E_{0}}\right)^{2}\right] \frac{\partial}{\partial E} .
$$

In the above we have used $E=p c$ and $E_{0}=p_{0} c$. With the modified position, momentum and energy one can show that $\left(c t^{\prime}\right) p^{\prime}-x^{\prime}\left(E^{\prime} / c\right)=(c t) p-x(E / c)$ i.e. the form of the generators for boosts is the same as in special relativity. Using the tanh modification of energy-momentum this can be seen as follows: $\left(c t^{\prime}\right) p^{\prime}=i \hbar c \cosh ^{2}\left(\frac{p}{p_{0}}\right) \frac{\partial}{\partial p}\left[p_{0} \tanh \left(\frac{p}{p_{0}}\right)\right]=$ $i \hbar c$ and for the standard operators one has $(c t) p=i \hbar c \frac{\partial}{\partial p}[p]=i \hbar c$. Thus $\left(c t^{\prime}\right) p^{\prime}=(c t) p$. A similar calculation applies to $x^{\prime}\left(E^{\prime} / c\right)$ showing that it is equivalent to $x(E / c)$. Finally 
for the arctan modification of energy-momentum the same type of calculation shows $\left(c t^{\prime}\right) p^{\prime}-x^{\prime}\left(E^{\prime} / c\right)=(c t) p-x(E / c)$.

The deviation of our modified operators from the standard operator is characterized by the parameter $p_{0}$. One can ask what kind of constraints can be placed on $p_{0}$ from observations. One of the common means to place bounds on phenomenological parameters (which give deviations from the standard energy-momentum and time-position operators) comes from the observation of photon dispersion. Since by construction our model was intended to evade these bounds, they do not constrain $p_{0}$. Bounds on models which modify/violate Lorentz symmetry can be given by other types of observations. These observations include pair production $\left(\gamma+\gamma_{b} \rightarrow e^{-}+e^{+}\right.$where $\gamma_{b}$ is a background photon), photon splitting $(\gamma \rightarrow \gamma+\gamma+\gamma)$, suppression of air showers, and photon decay $(\gamma \rightarrow$ $\left.e^{-}+e^{+}\right)$. Two recent articles which discuss these constraints and give observational limits on the modification/violation of Lorentz symmetry are [29] [30]. There is crucial dependence of these constraints on the order of the deviation in the relationship between energy and momentum. One can write a generic modified energy-momentum relationship as $E^{\prime 2}=p^{2}+K p^{n+2}$ which can be manipulated to yield $E^{\prime} \approx p\left(1+\frac{1}{2} K p^{n}\right)$. Here $K$ simply represents some constant which characterizes the particular model. The two common cases investigated for this modified energy-momentum relationship are $n=1$ and $n=2$. For the $n=1$ case the most stringent observational limit on the energy scale is $10^{22}$ $\mathrm{GeV}$ coming from photon decay. For $n=2$ the maximum limit on the energy scale is $10^{15} \mathrm{GeV}$ coming from photon splitting. For our proposed modified operators the resulting modified energy-momentum relationships, coming from (7) and (8), are to lowest non-trivial order $E^{\prime} \approx p c\left(1-\frac{1}{3} \frac{p^{2}}{p_{0}^{2}}\right)$ (to this order both tanh and arctan have the same expansion). Thus our modification corresponds to the $n=2$ case, which implies an energy bound of $10^{15} \mathrm{GeV}<E_{0}$. This in turn gives the bound on $p_{0}$ of $10^{15} \mathrm{GeV} / \mathrm{c}<p_{0}$.

A minimal distance was also obtained via a modified Heisenberg Uncertainty Principle (i.e. modification of the operators but not the commutator) in contrast to using the standard Generalized Uncertainty Principle (i.e. modification of the commutator and operators). We also introduced appropriately modified position operators in conjunction with the modified momenta from (5), which led to a minimal distance. This was accomplished without modifying the fundamental position -momentum commutation relationship. Many of the problems and issues associated with modifying the position-momentum commutator $[20,24]$ are avoided in this way. The approach to a minimal distance presented here also leads to a different relationship between the uncertainty in position and the uncertainty in momentum, as compared to the usual GUP approach, as can be seen by comparing Figs. 1 and Figs. 2.

Recent work [31] uses the Schwinger-Keldysh formalism to investigate the existence (or not) of a minimum geometrical length versus a minimum length scale. This work arrives at the conclusion that while there is no minimum geometrical length, there is a minimum length scale (identified as the Planck scale) beyond which scattering experiments become useless. Further reference [31] suggests, as does the present work, that quantum gravity effects can not be probed by looking for dispersion of photons propagating through spacetime.

Author Contributions: Conceptualization, J.C., M.B., J.L. and D.S.; formal analysis, J.C., M.B., J.L. and D.S.; investigation, J.C., M.B., J.L. and D.S.; writing-original draft preparation, J.C., M.B., J.L. and D.S. All authors have read and agreed to the published version of the manuscript.

Funding: This research received no external funding.

Acknowledgments: Some of the authors M.B. and D.S. acknowledge the FAMP seminar where some of the ideas for this paper were initially discussed. I, Joey Contreras, would also like to acknowledge my good friends Joseph Tilghman and Axel Fipps for always supporting me as well as my son Jayden Contreras who is the light of my world.

Conflicts of Interest: The authors declare no conflict of interest. 


\section{References}

1. L. J. Garay, Int. J. Mod. Phys. A 10, 145 (1995).

2. S. Das and E. Vagenas, Phys. Rev. Lett. 101, 221301 (2008).

3. P. Bosso, S. Das, and R. B. Mann, Phys. Lett. B 785, 498 (2018).

4. G. Amelino-Camelia, J. R. Ellis, N.E. Mavromatos, D. V. Nanopoulos, and S. Sarkar, Nature 393, 763 (1998).

5. G. Amelino-Camelia, Phys. Lett. B 510, 255 (2001).

6. G. Amelino-Camelia, Int.J.Mod.Phys.D 11, 35 (2002).

7. A.A. Abdo, et al., Nature 462, 331 (2009).

8. G. Amelino-Camelia, Nature, 418, 34 (2002).

9. G. Amelino-Camelia, Symmetry, 2,230 (2010).

10. J. Kowalski-Glikman, Phys. Lett. A. 286, 391 (2001).

11. J. Magueijo and L. Smolin, Phys. Rev. Lett., 88, 190403 (2001).

12. A. Kempf, G. Mangano and R. B. Mann, Phys. Rev. D 52, 1108 (1995).

13. M. Born and L. Infeld, Proc. Roy. Soc. A, 144, 425 (1934).

14. M. Maggiore, Phys. Lett. B 304, 65 (1993).

15. D. Amati, M. Ciafaloni, and G. Veneziano, Phys. Lett. B 216, 41 (1989).

16. D. Amati, M. Ciafaloni, and G. Veneziano, Phys. Lett. B 197, 81 (1987); Int. J. Mod. Phys. A, 03, 1615 (1988); Nucl. Phys. B 347, 550 (1990).

17. D.J. Gross and P.F. Mende, Phys. Lett. B 197, 129 (1987); Nucl. Phys. B 303, 407 (1988).

18. F. Scardigli, Phys. Lett. B, 452, 39 (1999).

19. R. J. Adler, D. I. Santiago, Mod. Phys. Lett. A 14, 1371 (1999).

20. M. Lake, " A New Approach to Generalised Uncertainty Relations", arXiv:2008.13183 [gr-qc].

21. M. Lake, M. Miller, and S. Liang, Universe 6, 56 (2020).

22. M. Lake, M. Miller, R. F. Ganardi, Z. Liu, S. Liang, and T. Paterek, Class. Quant. Grav. 36, 155012 (2019).

23. M. Lake, Ukr. J. Phys. 64, 1036 (2019)

24. G. Amelino-Camelia, Entropy 19, 400 (2017).

25. M. Bishop, J. Lee, and D. Singleton, Phys. Lett. B 802, 135209 (2020).

26. S. Ghosh and P. Pal, Phys.Rev.D 75, 105021 (2007)

27. K. Nozari, M. A. Gorji, V. Hosseinzadeh, and B. Vakili, Class. Quant. Grav. 33, 025009 (2016).

28. M. Bishop, E. Aiken, and D. Singleton, Phys. Rev. D 99, 026012 (2019).

29. H. Martínez-Huerta, R. G. Lang and V. de Souza, Symmetry, 12, 1232 (2020).

30. G. Rubtsov, P. Satunina and S. Sibiryakov. JCAP 05, 049 (2017).

31. R. Casadio and I. Kuntz, Eur. Phys. J. C 80, 958 (2020). 